\title{
Possibility of discrete beam propagation in chiral nematic liquid crystals
}

\author{
Michał Kwaśny, ${ }^{*}$ Urszula A. Laudyn, Paweł Jung, Mirosław A. Karpierz \\ Faculty of Physics, Warsaw University of Technology, Koszykowa 75, 00-662 Warszawa
}

Received December 9, 2009; accepted December 30, 2009; published December 31, 2009

\begin{abstract}
This work presents an experimental and theoretical analysis of beam propagation in multiple layers formed by chiral nematic liquid crystal. Depending on structure parameters light can be guided in a single layer or propagate in a matrix of coupled layers in a discrete way. It is also shown that the nonlinear behavior leads to creation of spatial solitons (nematicons) in both discrete and singlelayer configurations.
\end{abstract}

Nonlinear optical properties of liquid crystals attracted a lot of attention due to extremely large nonlinearity caused by the reorientational effect [1]. Among others, in liquid crystals spatial solitons, called nematicons are observed [2, 3]. Nematicons were observed for a laser power of a few milliwatts and at distances of a few millimetres.

The possibility to obtain nematicon propagation in chiral nematic liquid crystals (ChNLCs) has been also reported $[4,5]$. In this work we present the possibility to create discrete diffraction and discrete spatial solitons in layers created by ChNLCs film. We present experimental results showing the beam propagating independently in each layer and the numerical simulations showing the conditions necessary to obtain discrete diffraction. The configuration of the analyzed sample of chiral nematic film is presented in Fig. 1. The orientation of molecules at the boundaries is determined by the anchoring conditions, where the interaction with the cell walls introduces boundary orientation. The angle between molecules direction and $y$ axis is initially equal to $\Theta(x)=(2 \pi m / d) x$, where $m$ is an integer number, and $d$ is a sample thickness. Depending on incident light beam polarization we can identify two situations. Firstly, in the case of light polarized in the $x$ direction, the refractive index is constant across the sample. Secondly, in the case of light polarized in the $y$ direction, as a consequence of a helical structure, the refractive index varies across the sample from $n_{\mathrm{o}}$ (ordinary refractive index - for $\Theta=\pi / 2$ ) to $n_{\mathrm{e}}$ (extraordinary refractive index where $\Theta=0$ ). Along the $x$ axis (perpendicular to long molecular axes) layers with the same effective

"E-mail: mkwasny@if.pw.edu.pl refractive index can be identified, and light polarized in the $y$ direction propagates in the region where refractive index is the largest, i.e. where $\Theta \approx 0$.

Besides obtaining light propagation in the $y z$ plane, for certain conditions light can couple to the neighboring layers. Owing to the fact that the refractive index distribution changes along the $x$ direction, the analyzed cell can be treated as a matrix of waveguides structure with a periodically modified refraction index. In the proposed geometry it is possible to obtain conditions in which a conventional continuous diffraction is substituted by discrete one in the sense of discrete coupling between waveguides aside. The efficiency of getting light into nearby layers is inversely proportional to the distance between them and the modulation of the refractive index $\left(n_{\mathrm{o}}-n_{\mathrm{e}}\right)$.

In a nonlinear regime, as a consequence of lightinduced molecular reorientation and changes in effective refractive indices the beam is self-focused and finally the spatial soliton can be formed. In the $x z$ plane this self-focusing is treated as a decrease in the coupling between neighbouring layers, and the creation of a discrete spatial solitons. In the $y z$ plane the nonlinear behaviour is similar to the self-focusing and soliton creation in homogenous media.

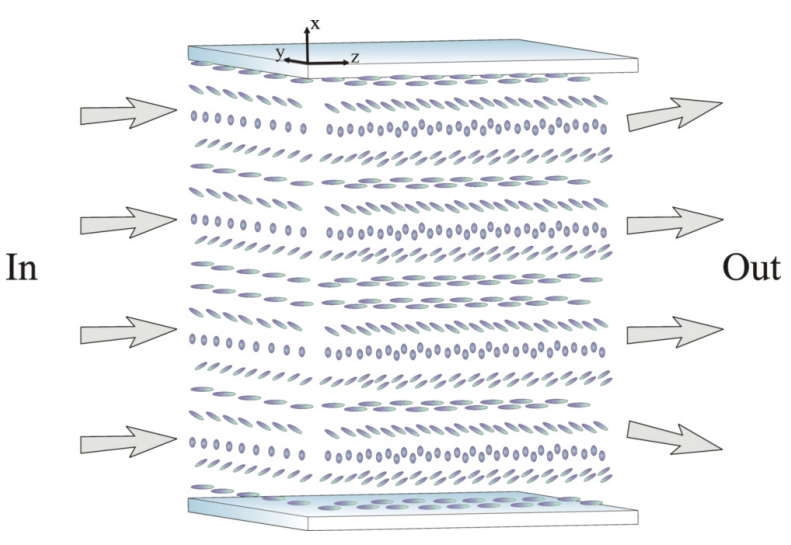

Figure 1. Configuration of ChNLCs cell. 
The typical configuration of our experimental setup has been presented in the previous paper [5]. As a light source we used a Ti: sapphire laser $(\lambda=793 \mathrm{~nm})$ or $\mathrm{Ar}^{+}$ laser $(\lambda=514 \mathrm{~nm})$. The beam propagated in the $z$ direction was focused in a cell by a microscope objective to the spot of about two micrometers in diameter. The analyzed sample was made of two glass plates glued together and filled through a capillary effect with 6CHBT (4-trans-4'-n-hexylcyclohexylisothiocyanatobenzene) doped with a chiral material. The pitch of the analyzed helix was about $25 \mu \mathrm{m}$ and the width of the cell was $50 \mu \mathrm{m}$. It means that is possible to mark out four layers where molecules are oriented in the same direction. For observation of a scattered light from the top of the cell a CCD camera was used. An additional camera placed at the back of the cell was used to control vertical position. However in the next steps it will be used for the observation of discrete light propagation.

For a low input power the beam broadens with a propagation distance because of diffraction. In a nonlinear regime (about 9 milliwatts of optical power) the self-focusing effect compensates the diffraction and the beam starts to form a nematicon, which does not change its shape during the propagation for a distance of about $1500 \mu \mathrm{m}$. The possibility of obtaining as many solitons as layers in the structure has been shown by moving the cell in the $x$ direction for the fixed polarization (TE, i.e. light polarized in the $y$ direction) with power high enough to induce molecular reorientation (Fig. 2). The vertical position of a cell has been estimated from the top of the cell (with an accuracy of about $2 \mu \mathrm{m}$ ). It means that in the position described as $\Delta x=0$ light falls on the top of the upper glass-plate. Those nematicons propagate independently in each layer, and the distance between them is about half the pitch value $(12.5 \mu \mathrm{m})$. The difference between ordinary and extraordinary refractive indices (birefringence of a medium) corresponds to $\Delta n=0.15$ at room temperature. In order to obtain a light coupling between layers with a coupling length of an order about tens/hundreds of microns, the conditions for amplitude of refractive index modulation and interval between the layers must be precisely selected. In the case of cholesteric liquid crystals presented above, no light coupling between the layers was observed. So we decided to perform measurements for cholesteric structure with different parameters. It has been characterized by lower birefringence (LB) equal to $\Delta n=0.06$ (at room temperature), and lower pitch value of $10 \mu \mathrm{m}$.

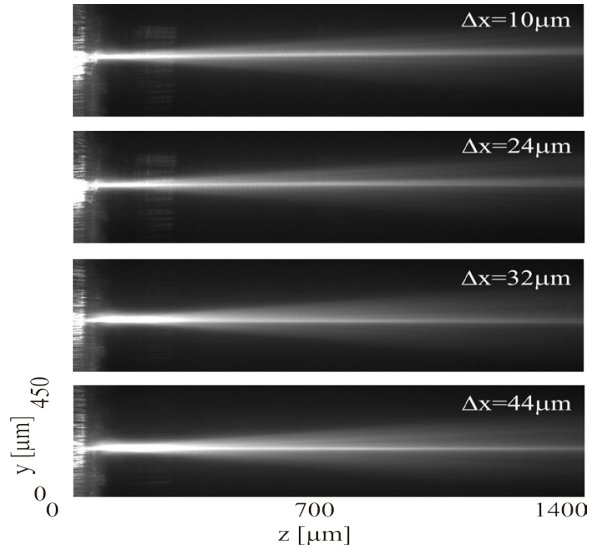

Figure 2. Experimental results of soliton propagation in different layers for different input beam positions (marked on photos).

A shorter pitch means higher density of guiding layers at the same cell width, and lower minimum power needed for molecular reorientation which causes solitary wave propagation. It was shown that soliton propagation in such a thin layer of chiral nematic is possible to obtain (Fig. 3). It is worth noting that together with a lower chirality pitch, the minimum power needed for nematicon propagation is also decreasing.

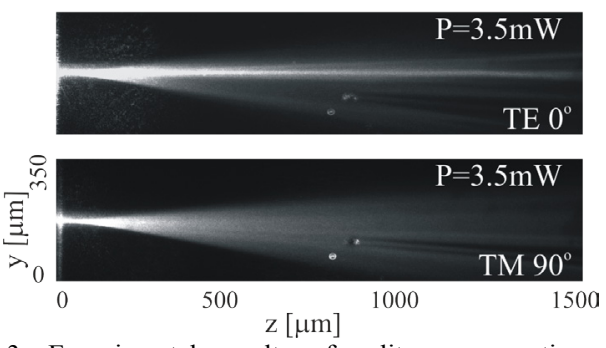

Figure 3. Experimental results of soliton propagation in low birefringent cholesteric LC for different light polarization.

For two types of periodic structures that correspond to ChNLCs structures investigated experimentally, $(1+1) \mathrm{D}$ BPM simulations have been run in order to check the possibilities of light coupling. The numerical calculations of light propagation have been performed for periodic structures with parameters that correspond to birefringence and pitch of analyzed chiral nematics. The beam waist is a fixed parameter equal to $2 \mu \mathrm{m}$.

The results obtained in linear regime are presented in Fig. 4. In the $y z$ plane light diffracts in a continuous way (Fig. $4 \mathrm{a}$ ) and propagation in the same plane in LB ChNLCs takes place in a similar way (that plane can be observed in a real experiment by a CCD camera). The cross-section of a cell along the $x z$ plane in high birefringent ChNLCs (Fig. 4b) shows that propagation occurs only in a thin layer. 
(a)

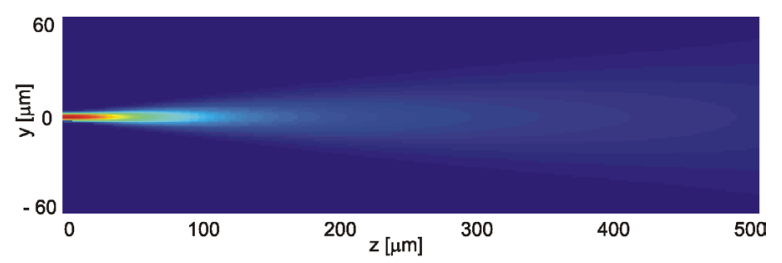

(b)

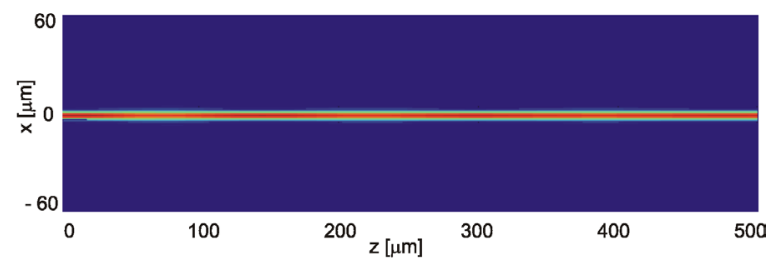

(c)

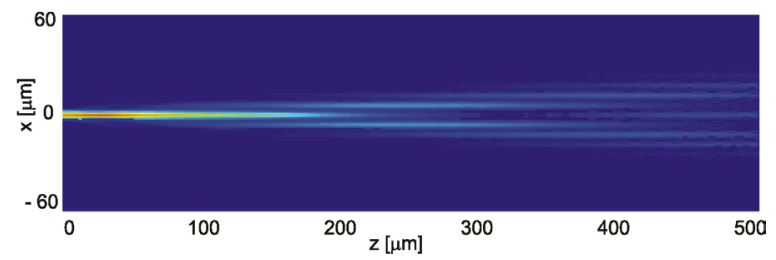

Figure 4. Numerical calculations of light propagation in $y z$ and $x z$ planes in ChNLCs for $\Delta n=0.15$, pitch $25 \mu m(\mathrm{a}, \mathrm{b})$ and in $x z$ plane for $\Delta n=0.06$, pitch $10 \mu m(\mathrm{c})$.

In Fig. (4c) calculations related to low birefringent cholesteric with a lower pitch are presented. A smaller pitch of analyzed chiral nematic means that the distance over which light is transferred from a layer to the neighboring one becomes shorter. For a pitch of $25 \mu m$ (Fig. 4b) the coupling length was higher than the length of the sample, and energy transfer between layers was not observed. When the distance between waveguides becomes shorter and modulation of refractive index was smaller light can couple between such layers and propagate in a discrete way.

In the nonlinear case as a consequence of the reorientational effect, in $y z$ plane the beam starts to propagate as a solitary wave and in $x z$ plane a discrete spatial soliton is formed (Fig. 5).

Concluding, the independent soliton propagation in layers with the same effective refractive index in ChNLCs has been demonstrated experimentally. (a)

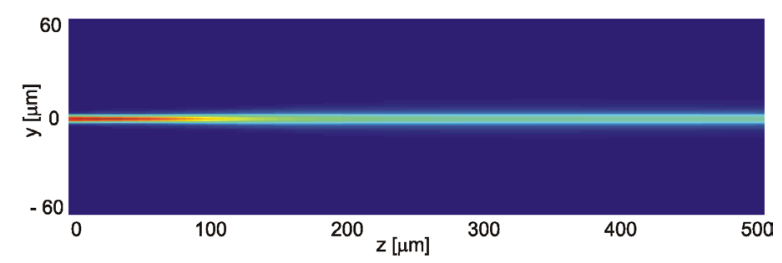

(b)

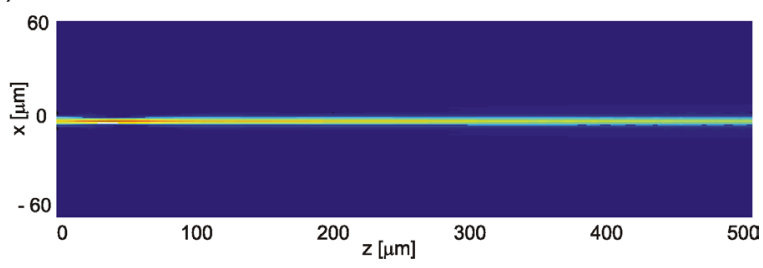

Figure 5. Numerical calculations of nonlinear light propagation in $y z$ (a) and $x z$ (b) planes in low birefringence ChNLCs, for $\Delta n=0.06$, pitch $10 \mu m, \Delta n_{N L}=0.108$.

Numerical simulations show that a coupling between that kind of waveguides and discrete light propagation may be attained in certain types of low birefringent cholesteric liquid crystals of a small pitch. This work was an introduction to discrete diffraction and discrete soliton observations and experiments with low birefringent cholesteric will be carried out in the near future. They seem very promising from the viewpoint of potential applications in all optical switchingsystems, because a very low power laser beam induces large nonlinear effects and properties of nematicons are very attractive for applications in low power all-optical steering and switching elements.

\section{References}

[1] I. C. Khoo, S. T. Wu, Optics and Nonlinear Optics of Liquid Crystals, World Scientific Publ., Singapore (1997)

[2] G. Assanto, M. Peccianti, C. Conti, Opt. Photon. News 14, 44 (2003)

[3] M. A. Karpierz, Soliton Driven Photonics, Boardman, A. D. Sukhorukov, A.P., (Eds.), p.41, Kluwer Academic Publishers: Dordrecht

[4] U. A. Laudyn, K. Jaworowicz, M. A. Karpierz, Mol. Cryst. Liq. Cryst. 489, 1563( 2008)

[5] U. A. Laudyn, M. Kwasny, M. A. Karpierz, Appl. Phys. Lett., 94, 091110 (2009) 